\title{
Surgically Implantable Long-term Antipsychotic Delivery Systems for the Treatment of Schizophrenia
}

\author{
Steven J. Siegel, Karen I. Winey, Raquel E. Gur, Robert H. Lenox, Warren B. Bilker, Debbie Ikeda, \\ Neel Gandhi, and Wen-Xiao Zhang
}

\begin{abstract}
Non-adherence with medication remains a major correctable cause for poor outcome in schizophrenia. We describe a surgically implantable preparation of haloperidol with the aim that patients will have superior outcomes with improved medication adherence from implants. In contrast to depot formulations, implantable pellets could last many months, providing symptomatic improvement for periods of time never before possible. Additionally, in the event of unacceptable side effects, implants could be removed, offering a degree of reversibility not available with depot formulations. A surgically-implantable formulation of haloperidol has been created using biodegradable polymers.
\end{abstract}

Implants have been characterized for in-vitro kinetics, as well as in-vivo bioactivity in rodents. Haloperidol implants demonstrate steady release of drug for 5 months. Animals treated with haloperidol implants display increased striatal D2 receptor expression as well as increased apomorphine stimulated locomotion. Surgically-implantable formulations are a viable approach to provide long-term delivery of antipsychotic medications to patients with psychotic disorders.

[Neuropsychopharmacology 26:817-823, 2002] (C) 2002 American College of Neuropsychopharmacology. Published by Elsevier Science Inc.
KEY WORDS: Antipsychotic; Compliance; Implant; Adherence; Schizophrenia; Treatment

While much research regarding the treatment of schizophrenia has focused on new pharmaceutical compounds, a major correctable cause for treatment resistance remains nonadherence with prescribed medication (Fenton et al. 1997; Kane 1985). Approximately 50\% of patients with

From the Division of Neuropsychiatry (SJS, REG, DI, W-XZ), Laboratory of Molecular Neuropsychopharmacology (SJS, RHL, DI, NG, W-XZ), Stanley Center for Experimental Therapeutics (SJS), Department of Psychiatry, Department of Materials Science and Engineering (KIW), and Department of Biostatistics and Epidemiology (WBB), University of Pennsylvania, Gates Building $10^{\text {th }}$ floor, 3400, Spruce Street, Philadelphia, PA 19104.

Address correspondence to: Steven Siegel, M.D., Ph.D. Tel.: (215)

573-0278, Fax: (215) 662-7903, E-mail: siegels@mail.med.upenn.edu

Received July 17, 2001; revised October 26, 2001; accepted November 26, 2001.

Online publication: $11 / 28 / 01$ at www.acnp.org/citations/ Npp112801212. schizophrenia, and other chronic medical conditions, are thought to be poorly adherent with prescribed medication (Young et al. 1986). Therapeutic failure secondary to nonadherence often results in deterioration in social function and more intensive interventions including rehospitalization, such that nonadherence is the most important predictor of rehospitalization in a state hospital population (Casper and Regan 1993). Additionally, consequences of medication nonadherence extend beyond the health of the patient. While the majority of people with schizophrenia do not engage in violent behavior, a subset of patients display aggression during periods of psychosis (Casper and Regan 1993; Lindqvist and Allebeck 1990; Mitchell 1999; Tam et al. 1996). Such incidents have spurred the revision of New York State's Mental Hygiene Law ("Kendra's Law") that enables mandatory, court-ordered outpatient treatment adherence for people with psychiatric conditions who "because of their illness, have great difficulty taking responsibility for their 
own care" (Bruno and Silver 1999). While such measures have become increasingly popular (Kohl 1999), they must wait until a patient demonstrates the consequences of nonadherence. Additionally, while Kendra's law is meant to be "compassionate, not punitive" (Bruno and Silver 1999), it assumes control of a patient's treatment decisions. Preferably, patients could retain autonomy and make treatment decisions during periods of health. Surgically implantable formulations would allow patients to make long-term medication treatment decisions during periods of health, rather than symptom exacerbation.

Other advantages of parenteral administration include lower dosing and steady state serum drug levels, with fewer resultant side effects. Increased bioavailability with less variation in absorption and no first pass metabolism also decrease variation in plasma levels between individuals for a given dose (Gerlach 1995; Kane 1985). These factors promote antipsychotic efficacy with reduced drug exposure and side effects. Finally, in the event of unacceptable side effects, such as neuroleptic malignant syndrome, an implant could be removed. This offers a degree of reversibility not available with depot formulations. The purpose of this study is to determine the feasibility of creating surgically implantable delivery systems for antipsychotic medication to help patients with schizophrenia with long-term adherence.

\section{METHODS}

\section{Implant Fabrication}

Implants were fabricated through solvent casting and compression-molding. Two polymers, $75 \%$ polylactide with $25 \%$ polyglycolide (75:25 PLGA) and $85 \%$ polylactide with $15 \%$ polyglycolide (85:15 PLGA) are presented in a combined system of release during a 5-month period. Each copolymer has a distinctive period of degradation that is determined by the ratio of lactide to glycolide and the molecular weight of the resulting molecule produced. An additional polymer of $100 \%$ polylactide (PLA) was used for in vivo testing in rats. All polymers (Alkermes Inc., Cincinnati, $\mathrm{OH}$ ) had an inherent viscosity of $0.66-0.80 \mathrm{DL} / \mathrm{g}$ in chloroform and a molecular weight distribution between 120-140 kD. Individual polymers and haloperidol (Sigma, St. Louis, MO) were dissolved in acetone and solvent cast at $60^{\circ} \mathrm{C}$ for $72 \mathrm{~h}$. Solvent-cast material was compression molded at $80^{\circ} \mathrm{C}$ and 25,000 psi to a final density of $1.1 \pm 0.05 \mathrm{~g} / \mathrm{cc}$ ).

\section{In-vitro Assay}

Individual implants were placed in 1 liter of phosphate buffered saline (PBS), pH 7.0 at $37^{\circ} \mathrm{C}$ on a shaker. Haloperidol concentration was measured by GCMS (National Medical Services, Willow Grove, PA). Each assay included negative controls of implants made of polymer alone and a $100 \mathrm{ng} / \mathrm{ml}$ haloperidol standard to assess stability of haloperidol in solution over time.

\section{Animals}

Implants were tested in rats (Harlan, Indianapolis, IN) $(\mathrm{n}=9)$ and mice (Jackson Labs, Bar Harbor, ME) ( $\mathrm{n}=$ 16). All animals were housed in an AAALAC accredited animal facility at the University of Pennsylvania. The Institutional Animal Care and Use Committee (IACUC) approved all protocols. Animals were maintained with a 12:12 light:dark cycle with all testing and procedures performed during the light cycle.

\section{Implantation/removal Surgery}

Mice and rats were anesthetized with ketamine/xylazine $(100 / 10 \mathrm{mg} / \mathrm{kg}$, i.p.). A $1-\mathrm{cm}$ scalpel incision was made in the skin on the dorsal aspect of the animal. The subdermal space was visualized with hemostats and a single implant was placed between dermis and muscle with forceps. The wound was then closed with a surgical staple. Four weeks after implantation, implants were localized by palpation and removal was performed with identical anesthesia and incision. Intact implants were easily removed with forceps.

\section{Behavioral Testing}

Sixteen C57bl/ 6 mice received implants made of 75:25 PLGA alone $(\mathrm{n}=8)$ or $75: 25$ with $20 \%$ haloperidol $(\mathrm{n}=$ 8 ) to assess the effects of implants on locomotion. Following three weeks of implantation, total distance traversed was assessed over a twenty-minute period. Implants were then removed and animals allowed to recover for $48 \mathrm{~h}$ prior to re-testing $20 \mathrm{~min}$ after apomorphine challenge ( $0.5 \mathrm{mg} / \mathrm{kg}$ i.p.) (Sigma, St Louis, MO).

\section{Western Blot}

Six Sprague-Dawley rats received implants made of PLA with $30-40 \%$ haloperidol. Three rats received implants of PLA alone. Implants remained in all animals for three months prior to removal. Seventy-two hours after implant removal, rats were sacrificed and brains rapidly removed, dissected into four regions (cortex, hippocampus, striatum and cerebellum) then frozen in liquid nitrogen. Western blots for quantitative analysis of D2 receptor protein were performed on striatum. Three concentrations of cortex protein from a single animal $(2.5,5$ and $10 \mu \mathrm{g})$ were run with all blots as an internal control to insure intensity of labeling was within linearity of quantitative software. Only those blots in which the density of samples was within the linear range of internal standards were used for analyses. 
Western blots were performed using polyclonal antibody WR-3526, raised against amino acids 272-282 of the D2 receptor protein (Research and Diagnostic Antibodies, Berkeley, Ca). Striata were homogenized in homogenization buffer (20 mM HEPES, 2mM EGTA, 1 $\mathrm{mM}$ PMSF, $2 \mu \mathrm{M}$ Aprotinin and 2mM DTE), followed by a 30-s sonication. Samples were centrifuged at $100,000 \times \mathrm{g}$ for $1 \mathrm{~h}$ at $4^{\circ} \mathrm{C}$. Pellets were resuspended and solubilized in homogenization buffer containing $0.1 \%$ Triton X-100. Proteins were extracted on ice for $45 \mathrm{~min}$ with occasional agitation. After extraction, proteins were centrifuged at $30,000 \mathrm{~g}$ for $30 \mathrm{~min}$ at $4^{\circ} \mathrm{C}$. Protein samples were prepared with $25 \% 4 x$ NuPAGE sample buffer plus $10 \%$ reducing agent (Invitrogen) and heat shocked at $70^{\circ} \mathrm{C}$ for $10 \mathrm{~min}$. Samples were separated on a $10 \%$ precasted mini-gel at 200 volts for $50 \mathrm{~min}$. Proteins were transferred to PVDF at 30 volts for $1 \mathrm{~h}$. Blots were blocked with $5 \%$ milk in TBS $(20 \mathrm{mM}$ Tris, $\mathrm{pH}=$ $7.5,0.5 \mathrm{M} \mathrm{NaCl})$, then washed for $15 \mathrm{~min}$. Blots were then incubated overnight with anti-D2-Receptor antibody, washed with TBS, and incubated with goat anti-rabbit horseradish peroxidase conjugate (BioRad, 1:4800) for $1 \mathrm{~h}$. Blots were then incubated with chemiluminescent substrate (Pierce) for $1 \mathrm{~min}$, wrapped with plastic and exposed to autoradiographic film.

\section{Quantification}

The intensity of each band was quantified using a densitometer model 7100 and quantitative analysis software (Bio Rad, Hercules, CA) and expressed as a ratio to the corresponding band in rat 1 to yield a ratio of intensity (rat $1=$ ratio of 1 ). All samples were processed simultaneously on a single blot to allow for quantitative comparisons between conditions.

\section{RESULTS}

\section{In-vitro}

Haloperidol concentration for two polymers, as well as combined values, are shown in Figure 1. Implants made of 75:25 PLGA with 40\% haloperidol resulted in a pattern of release characterized by an initial phase of slow release (approximately $0.29 \%$ / day / implant) from 0 to 28 days. A second phase of more rapid release occurred between 28 and 84 days (approximately $1.28 \%$ / day / implant). Implants made with 75:25 PLGA released $50 \%$ of the total haloperidol load in 66 days (Figure 1, panel A). Implants made of 85:15 PLGA with $40 \%$ haloperidol displayed a similar pattern of release with a phase of slow release (approximately $0.26 \%$ / day / implant) from 0 to 56 days. A second phase of more rapid release occurred between 56 and 140 days $(0.95 \%$ / day / implant). Implants made from 85:15 PLGA released half of the haloperi- dol load in 88 days (Figure 1, panel B). Release from the theoretical composite system of 75:25 and 85:15 PLGA, $40 \%$ haloperidol, shows an early phase from 0 to 28 days with an average of $0.34 \%$ /day and more rapid release between 28 to 140 days with $0.82 \%$ /day. The combined system of 75:25 and 85:15 PLGA demonstrates a period of 78 days to release half of the total haloperidol load (Figure 1, panel C). The value for the positive control solution $(100 \mathrm{ng} / \mathrm{ml})$ remained stable throughout the period of 154 days $(0$ days $=106,154$ days $=100$, mean \pm sd throughout study interval $=$ $106 \pm 11 \mathrm{ng} / \mathrm{ml})$.

\section{In-vivo}

Locomotor Activity. Locomotor activity for mice that received either haloperidol or blank polymer implants is demonstrated in Figure 2. All animals were tested three weeks after receiving implants made of 75:25 PLGA alone or 75:25 PLGA with $20 \%$ haloperidol. Baseline locomotor activity was measured for twenty minutes. Animals with control implants traveled a mean of $12223 \pm 433 \mathrm{~cm}$, while those with haloperidol containing implants traveled an average of $7664 \pm 450$ $\mathrm{cm}$. Thus, mice with haloperidol implants traveled significantly less distance than controls $(p<.01)$. Implants were then removed and all animals were allowed to recover for $48 \mathrm{~h}$. One animal with a control implant died from anesthetic during removal surgery. Forty-eight hours after removal of implants, animals received apomorphine $0.5 \mathrm{mg} / \mathrm{kg}$ i.p. twenty minute prior to locomotor testing, which has been shown to increase locomotor activity in mice (Ninan and Kulkarni 1999). After apomorphine challenge, animals that had control implants traveled a mean of $4721 \pm$ $476 \mathrm{~cm}$, while those with haloperidol containing implants traveled an average of $8531 \pm 2536 \mathrm{~cm}$. Therefore, following removal of implants and exposure to apomorphine, mice that had haloperidol implants traveled more distance than control mice $(p<.01)$.

\section{Western Blots}

Western blots of striatal membranes from all rats revealed a band at an apparent molecular weight of approximately $50 \mathrm{kD}$ (Figure 3), corresponding to the predicted molecular weight of the full-length D2 receptor protein (Expert Protein Analysis System, Swiss Institute of Bioinformatics, http://www.expasy.ch/) (Bunzow et al. 1988). Mean optical density of bands was quantified relative to the corresponding band in lane 1 (haloperidol treated rat). Results based on three blots yielded a mean \pm SD relative density for haloperidol implant treated rats of $0.90 \pm 0.07$ for the $50 \mathrm{kD}$ band, while the mean relative density for control rats was $0.64 \pm 0.02$ $(p<.001,2$-tail $t$-test $)$. 

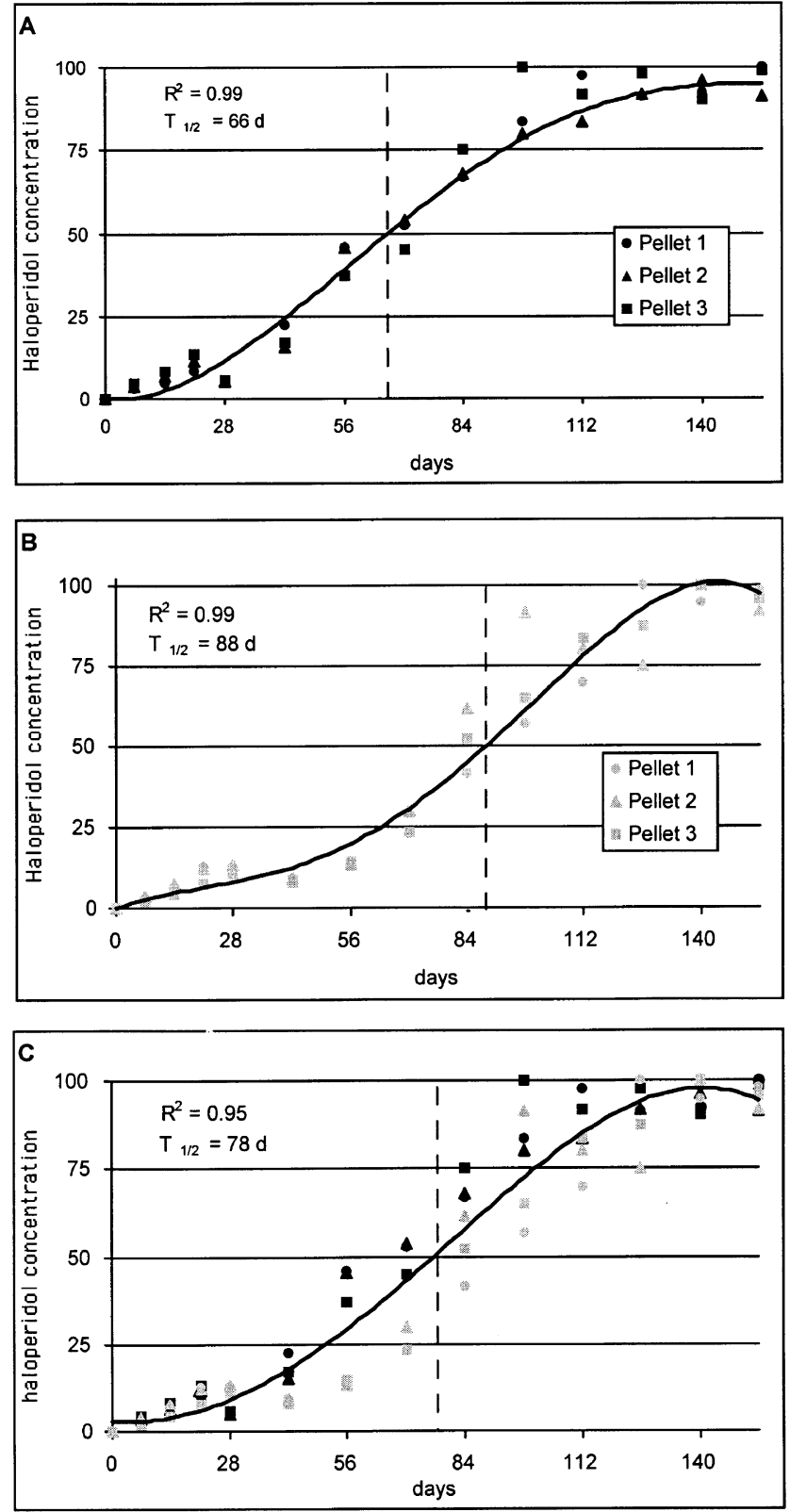

Figure 1. In vitro haloperidol release from polymer based implant system. Individual implants are placed in phosphate buffered saline, $\mathrm{pH} 7$, at $37^{\circ} \mathrm{C}$ on a shaker. Samples of buffer are drawn at weekly / biweekly intervals and evaluated with GCMS for haloperidol concentration. Cumulative release is expressed as the percent of total drug load measured in buffer solution at each time point. A, B: Cumulative haloperidol concentration from 75:25 and 85:15 PLGA polymers. Each graph displays data from three implants (circle, square and triangle) with a $4^{\text {th }}$ degree polynomial average line for the three values. A: Implants made from 75:25 PLGA: $40 \%$ haloperidol have a slow release lag phase of approximately one month before entering rapid release with a period of 66 days to release half of the total drug load. B: Implants from 85:15 PLGA: 40\% haloperidol have a 2-month slow release lag phase with rapid release between 2 and 5 months and a period of 88 days to release half of the total drug load. Drug load for each prototype implants is approx-

\section{DISCUSSION}

Despite advances in side-effect profiles for antipsychotic medication, adherence remains a major determinant of treatment outcome. To address this important clinical variable, we propose the use of surgically implantable formulations of antipsychotic medicine to improve adherence among a subset of people with schizophrenia. This study demonstrates a potential design for antipsychotic implants based on PLGA copolymers of variable compositions. This design is capable of providing delivery of clinically appropriate doses of biologically active haloperidol for five months. As release from a combined system of PLGA implants provides $0.34 \%$ / day during the first month and $0.82 \%$ / day during months 2-5 post implantation, a drug load of $200 \mathrm{mg}$ would yield $0.68 \mathrm{mg} / \mathrm{d}=20 \mathrm{mg}$ during the first month and $1.6 \mathrm{mg} / \mathrm{d}$ or $49 \mathrm{mg} / \mathrm{month}$ during months $2-5$. At a drug load of $40 \%$, as in the current system, this would require approximately a $500 \mathrm{mg}$ implant for five months. Thus, a two-implant system ( $250 \mathrm{mg}$ each) would require circular pellets to be approximately $2 \mathrm{~mm}$ thick by $12 \mathrm{~mm}$ in diameter, which is likely to be easily tolerated. Similarly, the procedure for implantation (and removal if necessary) could be performed in approximately $15 \mathrm{~min}$ with local anesthetic, adding to the likelihood that the surgery would be easily tolerated by patients. As described for rodents, this procedure would include a 1-2 centimeter scalpel incision, followed by clearing of a small subdermal space with hemostats and placement of the implants below the skin. The surgical wound would then be closed with sutures.

However, use of antipsychotic implants would require safeguards to ensure that patients are able to provide informed consent. While issues of informed consent for treatment of psychosis are not unique to surgical implants, the invasive nature of the procedure requires careful attention to protect patient autonomy (Brabbins et al. 1996; Carpenter and Conley 1999; Wong et al. 2000). Consistent with this goal, one advantage of implantable antipsychotic preparations would be that patients could make treatment decisions during periods of relative health. Poor insight and psychotic content during exacerbations of illness may impair capacity for making treatment decisions. When patients refuse treatment under such circumstances, patient autonomy is placed at odds with appropriate and indicated care. Furthermore, nonadherence with indicated treatment during periods of impaired capacity secondary to symptom exacerbation may lead to implementation of invol-

imately $22 \mathrm{mg}$. C: Cumulative release from a hypothetical two-polymer system over a period of 154 days. Each point represents a pellet shown in A (75:25 PLGA, black) or B (85:15 PLGA, gray). The combined system is characterized by a period of 78 days to release half of the total drug load. 


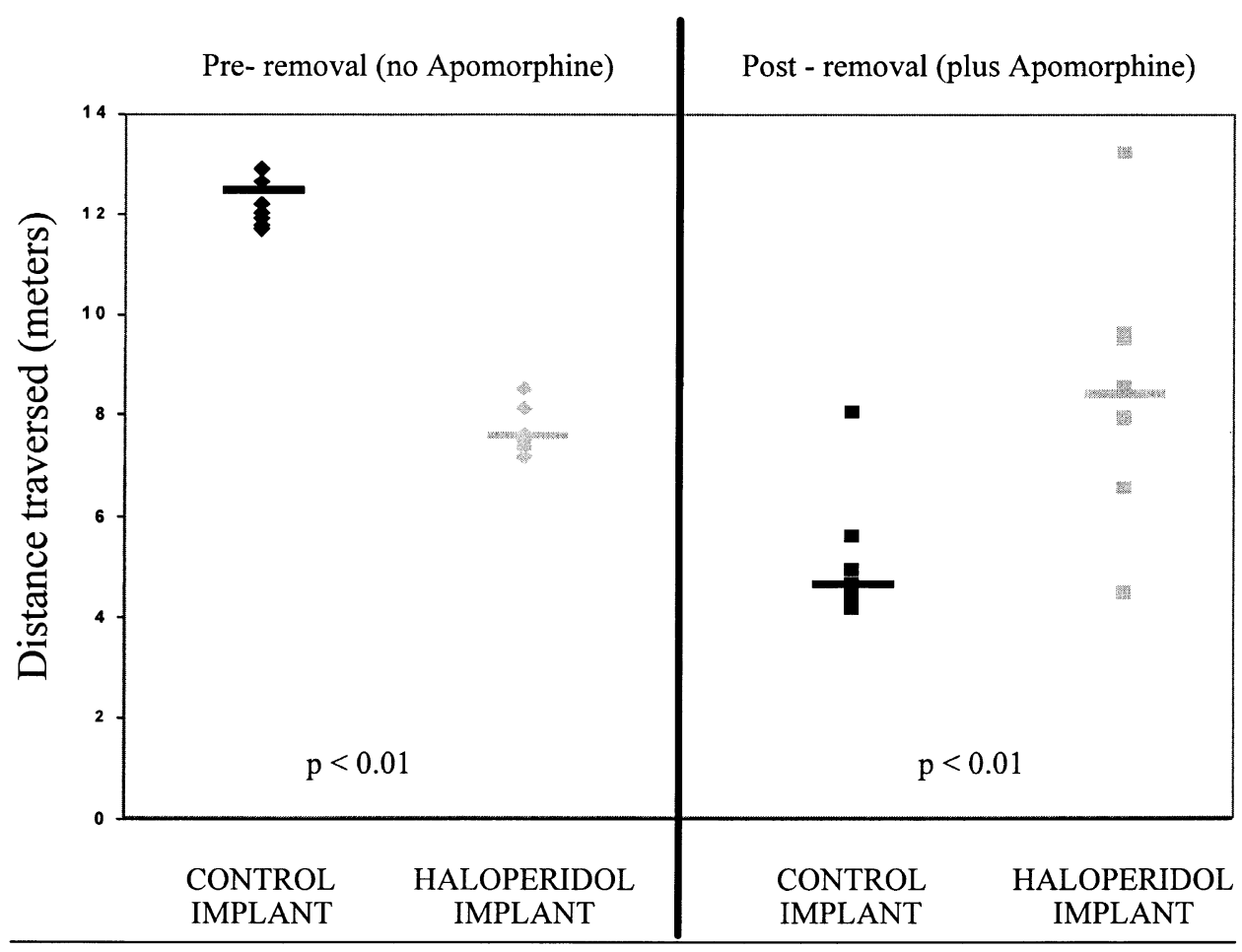

Figure 2. Locomotor activity (total distance in meters over twenty minutes) was tested prior to implant removal and then again $48 \mathrm{~h}$ following implant removal with the addition of apomorphine $(0.5 \mathrm{mg} / \mathrm{kg})$. Results indicate that mice with implants containing haloperidol display decreased activity prior to removal, and increased activity $48 \mathrm{~h}$ following removal of implants after exposure to apomorphine, consistent with striatal D2 blockade and upregulation in animals with haloperidol from implants. Therefore, haloperidol appears to retain bioactivity when released from polymer implants. black $=$ mice with control implants $(n=8)$, gray = mice with haloperidol implants $(n=8)$. horizontal lines indicate group mean.

untary inpatient or outpatient commitment. However, the majority of patients with psychotic disorders are able to achieve periods of symptom remission following treatment with medication. During such periods, people could choose to accept surgical preparations that would ensure that they receive medication during the subsequent five months. Thus, the patient retains the autonomy of treatment decisions by capitalizing on the ability to make those decisions in health.

Adverse reactions and side effects are additional concerns when considering implantation of a multi-month supply of medication. To minimize these risks, patients should be stabilized on oral, then depot formulations of the drug in use. However, if an adverse event occurs, implants retain a degree of reversibility not available with depot preparations. Since implants serve as a reservoir for the unused portion of the dose, the remaining supply can be removed with a simple procedure. Specifically, the variable polymer design capitalizes upon inclusion of polymers with both shorter and longer delivery times that retain superior coherence during slow release phase enabling them to be easily removed. Therefore, a portion of the implants would sit "dormant", while others enter into rapid release phase. While the current preparation includes only two polymers with release during five months, other polymers are capable of releasing antipsychotic agents for far longer periods with slow release phases greater than six months (Siegel et al. 2000). By combining a series of staged polymers to bridge successive periods of release, this design could extend the release of medication for a year or longer.

Factors other than release kinetics are required for a long-term delivery system to work. The drug must retain bioactivity following processing and sequestration within the polymer matrix. Haloperidol has been previously demonstrated to retain bioactivity (Kohler et al. 1994) and efficacy through parenteral administration (Beresford and Ward 1987; Chouinard et al. 1989). This was supported in the current study with evidence for D2 receptor upregulation following implantation of haloperidol-containing pellets in rats and mice. While parenteral activity is a requirement for implant suitability, the current approach is not limited to haloperidol. In fact, PLGA has been employed in injectable depot microsphere preparations with other agents including chlorpromazine (Suzuki and Price 1985) and risperidone (http://www.alkermes.com/index_news.html). 


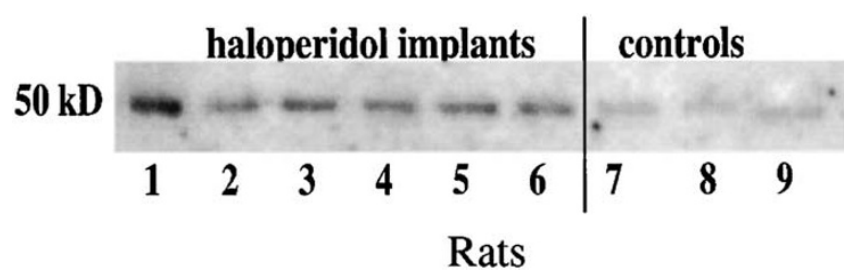

Figure 3. Western blot of D2 receptor protein in striatal membranes from rats treated with haloperidol-PLA or PLA alone implants for three months. A $50 \mathrm{kD}$ band, corresponding to the predicted apparent molecular weight of the full length D2 receptor, is labeled with polyclonal antibody WR3526 (Research and Diagnostic antibodies, Berkeley, CA), raised against amino acids 272-282. Note that lanes 1-6 contain striatum from animals that were exposed to haloperidol implants, while lanes 7-9 contain striatum from control animals. Mean optical density of bands was quantified relative to the corresponding band in lane 1 (haloperidol treated rat). Results based on three blots yielded a mean \pm SD relative density for haloperidol implant treated rats was $0.90 \pm 0.07$ for the $50 \mathrm{kD}$ band. The mean $\pm \mathrm{SD}$ relative density for control rats was $0.64 \pm 0.02$ for the $50 \mathrm{kD}$ band $(p=.0004$ based on a 2 -tail $t$-test comparison with haloperidol treated group).

While it is possible that the long-term implantable approach advocated here could be extended to these agents, additional drug characteristics must be considered in moving from depot (microspheres), which are dosed approximately every two weeks, to implantable pellets lasting many months to years: (1) the volume of material required for an extended multi-month supply. Since the amount of drug required is proportional to the potency of the agent, high potency agents with relatively low dose requirements like haloperidol and risperidone are ideally suited for implantation. (2) stability during both processing of implants and sequestration in the body at $37^{\circ} \mathrm{C}$. The current study demonstrates haloperidol's stability up to five months in physiological saline at body temperature. (3) avoiding local tissue reaction at the injection site. In both mice and rats, the current preparation was easily removed without signs of capsule formation, and drug effects were observed at one and three months. However, further testing in additional species and humans will be needed to confirm lack of local irritation, as well as variability in delivery kinetics introduced by biological factors such as body temperature and body mass index.

In summary, we advocate use of long-term surgically implantable formulations of antipsychotic medication as a mechanism to improve adherence in a subset of patients with psychotic disorders. This study demonstrates the feasibility of creating such a system with haloperidol and variable PLGA copolymers for staged release over five months. Future directions include extension to longer delivery intervals, as well as incorpo- ration of other medications for treatment of psychosis. Additionally, implantable formulations can be applied to many disorders where long-term medication is indicated, including affective illness and non-psychiatric medical disorders.

\section{ACKNOWLEDGMENTS}

We thank Drs. E. Fuller Torrey and Michael Knable for their invaluable support and encouragement of this work. We also thank Dr. Hank Kung and Dr. Mei-Ping Kung for helpful discussions.

All work was done within the Departments of Psychiatry and Material Science and Engineering, University of Pennsylvania, Philadelphia, PA 19104

This work was supported by The Theodore and Vada Stanley Foundation Research Award (SJS) and the National Alliance for Research on Schizophrenia and Depression (NARSAD) Young Investigator Award (SJS).

\section{REFERENCES}

Beresford R, Ward A (1987): Haloperidol decanoate. A preliminary review of its pharmacodynamic and pharmacokinetic properties and therapeutic use in psychosis. Drugs 33:31-49

Brabbins C, Butler J, Bentall R (1996): Consent to neuroleptic medication for schizophrenia: clinical, ethical and legal issues [see comments]. Br J Psychiatry 168:540-544

Bruno S, Silver S (1999): Chapter 408. New York State Constitution.

Bunzow JR, Van Tol HH, Grandy DK, Albert P, Salon J, Christie M, Machida CA, Neve KA, Civelli O (1988): Cloning and expression of a rat D2 dopamine receptor cDNA [see comments]. Nature 336:783-787

Carpenter WT, Conley RR (1999): Sense and nonsense: an essay on schizophrenia research ethics. Schizophr Res 35:219-25

Casper ES, Regan JR (1993): Reasons for admission among six profile subgroups of recidivists of inpatient services. Can J Psychiatry 38:657-661

Chouinard G, Annable L, Campbell W (1989): A randomized clinical trial of haloperidol decanoate and fluphenazine. J Clin Psychopharmacol 9:247-253

Fenton WS, Blyler CR, Heinssen RK (1997): Determinants of medication compliance in schizophrenia: empirical and clinical findings. Schizophr Bull 23:637-651

Gerlach J (1995): Depot neuroleptics in relapse prevention: advantages and disadvantages. Int Clin Psychopharmacol 9(Suppl 5):17-20

Kane J (1985): Compliance issues in outpatient treatment. J Clin Psychopharmacol 5:22s-27s

Kohl M (1999): New York state moves toward involuntary residential commitment of the mentally ill, Psychiatric Times, Vol 16, pp 30-32

Kohler U, Schroder H, Augustin W, Sabel BA (1994): A new animal model of dopamine supersensitivity using s.c. 
implantation of haloperidol releasing polymers. Neurosci Lett 170:99-102

Lindqvist P, Allebeck P (1990): Schizophrenia and crime. A longitudinal follow-up of 644 schizophrenics in Stockholm. Br J Psychiatry 157:345-350

Mitchell EW (1999): Does psychiatric disorder affect the likelihood of violent offending? A critique of the major findings. Med Sci Law 39:23-30

Ninan I, Kulkarni SK (1999): Differential effects of olanzapine at dopamine D1 and D2 receptors in dopamine depleted animals. Psychopharmacology (Berl) 142:175-181

Siegel SJ, Winey K, Lenox RH, Gur RE, Ikeda D, Ghandi N, Zhang W (2000): A surgically-implantable long term antipsychotic delivery system. ACNP meeting
Suzuki K, Price JC (1985): Microencapsulation and dissolution properties of a neuroleptic in a biodegradable polymer, poly(d,1-lactide). J Pharm Sci 74:21-24

Tam E, Engelsmann F, Fugere R (1996): Patterns of violent incidents by patients in a general hospital psychiatric facility. Psychiatr Serv 47:86-88

Wong JG, Clare CH, Holland AJ, Watson PC, Gunn M (2000): The capacity of people with a 'mental disability' to make a health care decision. Psychol Med 30:2 95-306

Young JL, Zonana HV, Shepler L (1986): Medication noncompliance in schizophrenia: codification and update. Bull Am Acad Psychiatry Law 14:105-122 